\title{
T cell responses to human platelet antigen-1a involve a unique form of indirect allorecognition
}

\author{
Maria Therese Ahlen, ${ }^{1,2}$ Anne Husebekk, ${ }^{2}$ Ida Løken Killie, ${ }^{2}$ Bjørn Skogen,,${ }^{1,2}$ and Tor Brynjar Stuge ${ }^{2}$ \\ 'Division of Diagnostic Services, Department of Laboratory Medicine, University Hospital of North Norway, Tromsø, \\ Norway. ${ }^{2}$ Immunology Research Group, Department of Medical Biology, University of Tromsø - \\ The Arctic University of Norway, Tromsø, Norway.
}

Fetal and neonatal alloimmune thrombocytopenia (FNAIT) is a pregnancy-related condition caused by maternal antibodies binding an alloantigen on fetal platelets. In most cases the alloantigen is formed by a single amino acid, integrin $\beta 3$ Leu33, referred to as human platelet antigen-1a (HPA-1a). Production of anti-HPA-1a antibodies likely depends on CD4+ $T$ cells that recognize the same alloantigen in complex with the HLA-DRA/DRB3*01:01 molecule. While this complex is well characterized, $T$ cell recognition of it is not. Here, to examine the nature of antigen recognition by HPA-1a-specific T cells, we assayed native and synthetic variants of the integrin $\beta 3$ peptide antigen for binding to DRA/DRB3*01:01-positive antigen-presenting cells and for $T$ cell activation. We found that HPA-1a-specific $T$ cells recognize non-allogeneic integrin $\beta 3$ residues anchored to DRA/DRB3*01:01 by the allogeneic Leu 33 , which itself is not directly recognized by these $\mathrm{T}$ cells. Furthermore, these $\mathrm{T}$ cell responses are diverse, with different $\mathrm{T}$ cells depending on different residues for recognition. This represents a unique form of indirect allorecognition in which a non-allogeneic peptide sequence becomes immunogenic by stable anchoring to MHC by an allogeneic residue.

Conflict of interest: The authors have declared that no conflict of interest exists.

Submitted: January 15, 2016 Accepted: August 4, 2016 Published: September 8, 2016

Reference information: JCI Insight. 2016;1(14):e86558. doi:10.1172/jici.insight.86558.

\section{Introduction}

Fetal and neonatal alloimmune thrombocytopenia (FNAIT) is a condition most commonly caused by maternal antibodies against human platelet antigen-1a (HPA-1a), transferred over the placenta during pregnancy. This platelet alloantigen is defined by a single amino acid difference at residue 33 of the integrin $\beta 3$ protein (1). About $\%$ of individuals of European descent are HPA-1b (Pro33) homozygous (HPA$1 \mathrm{bb})$. Women with this phenotype may become HPA-1a immunized in connection with pregnancy when the fetus has a paternally inherited HPA-1a allotype. In addition, the vast majority of HPA-1a-immunized women carry the MHC class II allele $H L A-D R B 3^{*} 01: 01$ (2-4), while its frequency in the general population is less than $30 \%$ (M.T. Ahlen, unpublished observations; refs. 5, 6). This strong association suggests that anti-HPA-1a antibody production is supported by $\mathrm{T}$ cell responses dependent on this allele. Indeed, HPA-1a-specific $H L A-D R B 3^{*} 01: 01$-restricted $\mathrm{CD}^{+} \mathrm{T}$ cells have been isolated from women who have given birth to children affected by FNAIT $(7,8)$. These T cells specifically recognize integrin $\beta 3$ peptides that include leucine at residue 33 (HPA-1a peptides). Characterization of the DRA/DRB3*01:01 peptide-binding motif demonstrated that HPA-1a peptides bind stably while integrin $\beta 3$ peptides with proline at residue 33 (HPA-1b peptides) bind poorly in comparison (9). The same study identified Leu33 as one of three anchor residues required for stable binding of integrin $\beta 3$ peptide to the DRA/DRB3*01:01 molecule. Crystallization of integrin $\beta 3$ peptide in complex with the DRA/DRB3*01:01 molecule revealed that Leu33 mediated peptide anchoring is facilitated by a hydrophobic pocket (P9) accommodating the hydrophobic leucine side-chain (10). This structural accommodation largely explains the basis for the observed differences in binding to this MHC molecule between Leu33 and Pro33 peptides (9). It also suggests that the Leu33 side-chain will be buried in the peptide-binding groove of the MHC molecule. This raises the question as to what HPA-1a-specific $\mathrm{T}$ cell clones actually recognize. In the present study, we address this question by examination of peptide recognition by 14 different HPA-1a-specific T cell clones isolated from alloimmunized women. 


\section{Results}

To determine the nature of antigen recognition associated with FNAIT, a panel of 14 different HPA-1aspecific clonal T cell lines - each expressing unique TCR $\alpha$ and TCR $\beta$ chains (Table 1) - were established from 3 different women in long-term cultures by stimulation with HPA-1a peptide (L33 peptide 20-mer), defined here as integrin $\beta 3$ residues 19-38, with the allogeneic leucine at position 33 (Leu33; Table 2). In our general scheme to achieve this, as detailed in subsequent sections, each $\mathrm{T}$ cell clone was assessed for activation by coculture with the DRA/DRB3*01:01-positive B-lymphoblastoid cell line (B-LCL) D4BL4 (Table 3) pulsed with 12-mer peptides (integrin $\beta 3$ residues $24-35$ ): HPA-1a peptide or peptides with various amino acid substitutions (Table 2). In parallel, the relative binding efficiency to antigen presenting cells (APCs) of these peptides - extended with a 15-mer N-terminally biotinylated linker — was determined by flow cytometry with a fluorescent streptavidin conjugate.

Peptide-binding assay. In order to determine the relationship between peptide binding and $\mathrm{T}$ cell activation, we developed a peptide-binding assay. The functionality of this assay was demonstrated by comparing the peptide-binding capacity of various in-house and MHC homozygous cell lines matched for MHC class II expression (Figure 1 and Table 3). Of these cell lines (Table 3), L33 peptide bound markedly better to DRA/DRB3*01:01-positive cells D4BL4 and STEINLIN than to cells expressing closely related allelic variants DRB3*02:02 (DUCAF) and DRB3*03:01 (EMJ). Furthermore, binding of the autologous HPA-1b peptide (P33; Table 2) was considerably lower on DRA/DRB3*01:01-positive APCs, as was binding of this peptide to DRB3*01:01-negative DUCAF and EMJ. This suggests that L33 peptide-binding to STEINLIN and D4BL4 is due to the DRA/DRB3*01:01 molecule, since binding to other MHC class II molecules can be ruled out due to matched MHC class II alleles on DUCAF and EMJ (Table 3). Slightly higher peptide-binding intensities were consistently detected with STEINLIN compared with D4BL4 (Figure 1); while STEINLIN is homozygous for DRB3 and carries $2 D R B 3^{*} 01: 01$ alleles, D4BL4 is heterozygous and carries only 1 copy (determined by a quantitative assay for genomic $D R B 3^{*} 01: 01$; M.T. Ahlen, unpublished observations). Although both STEINLIN and D4BL4 can bind exogenous peptide and activate HPA-1aspecific T cells, STEINLIN is HPA-1a positive and expresses endogenous integrin $\beta 3$, which can result in weak activation of the most sensitive T cell clones. Therefore, D4BL4 cells, which are HPA-1a negative, were used for peptide stimulation of T cells.

HPA-1a-specific T cells do not exclusively recognize integrin $\beta 3$ residue Leu33. With the above tools, we examined the fine specificity of antigen recognition by HPA-1a-specific T cells. First, to determine whether the Leu33 residue is directly recognized by HPA-1a-specific $\mathrm{T}$ cells, we examined $\mathrm{T}$ cell activation and peptide-binding to APCs with peptides in which Leu33 was substituted with small hydrophobic amino acids valine (V33) or isoleucine (I33), which like leucine are predicted to conform to the hydrophobic P9 pocket of DRA/DRB3*01:01. For 13 of 14 clones, stimulation with I33 and V33 peptides resulted in similar T cell activation compared with L33 peptide; one clone did not recognize either I33 or V33 peptides (Figure 2, A-C and Table 4). The magnitude of $\mathrm{T}$ cell activation was proportional to the efficiency of binding of peptides; as expected, I33 and V33 peptides bound to DRB3*01:01-positive APCs at levels similar to L33 peptide and at higher levels compared with peptides with a non-hydrophobic anchor at this position (P33, R33, and E33; Figure 2D, upper half). Differences in binding between these peptides were even more pronounced in the presence of adamantane ethanol (AdEtOH) as an MHC-loading enhancer (MLE) (11); Figure 2D, lower half). In contrast, peptides substituted at position 33 with charged amino acid arginine (R33) or glutamic acid (E33), or with proline (P33; HPA-1b) — none of which conform to the hydrophobic P9 pocket - bound considerably less to the APCs and did not activate the T cell clones. A similar low level of binding to DRA/DRB3*01:01-positive APCs was observed with peptides lacking one each of the other 2 of 3 anchor residues for binding to this MHC molecule (peptides A25 and A28 in Figure 3). These observations suggest that most HPA-1a-specific T cells do not exclusively recognize the allogeneic Leu33 residue, since substitution with other small hydrophobic residues did not diminish $\mathrm{T}$ cell activation.

HPA-1a-specific T cells recognize non-allogeneic residues in the HPA-1 a peptide. Since antigen recognition by most HPA-1a-specific T cells was determined not to involve specific recognition of the allogeneic integrin $\beta 3$ Leu33 residue, we reasoned that residues in the HPA-1a peptide recognized by these T cells must be non-allogeneic. To examine this, we tested various peptides, each representing single or multiple amino acid modifications of HPA-1a peptide (12-mer peptides, Table 2), for binding to APCs (Figure 3) and for activation of HPA-1a-specific T cells by peptide-pulsed APCs (Table 4). In these and following experiments, an MLE was used to facilitate peptide pulsing. 
Table 1. The TCR sequence rearrangements of T cell clones in this study (IMGT nomenclature)

\begin{tabular}{|c|c|c|c|c|c|c|c|}
\hline \multirow[b]{2}{*}{ Clone } & \multicolumn{3}{|c|}{ TCR $\alpha$} & \multicolumn{4}{|c|}{ TCR $\beta$} \\
\hline & TRAV & TRAJ & Sequence & TRAV & TRBD & TRBJ & Sequence \\
\hline D7T4 ${ }^{A}$ & $17^{*} 01$ & $57^{*} 01$ & CATFAQGGSEKLVF & $12-3^{*} 01 / 12-4^{*} 01$ & $1^{*} 01$ & $2-1^{*} 01$ & CASRGTSLYNEQFF \\
\hline D8T7 & $26-1^{*} 01 / 02$ & $23^{*} 01$ & CIVRLSYYNQGGKLIF & $9^{*} 01$ & $1^{*} 01$ & $1-3^{*} 01$ & CASAQGFGNTIYF \\
\hline D8T64 & $8-3^{*} 01 / 02$ & $23^{*} 01$ & CAVSNNQQGGKLIF & $11-2^{*} 01$ & $1^{*} 01$ & $2-5^{*} 01$ & CASSFRHGETQYF \\
\hline D8T48 & Not identified & & & $7-2^{*} 01 /{ }^{*} 04$ & $1^{*} 01$ & $1-2^{*} 01$ & CASSSDILRGYTF \\
\hline D8T102 & $8-4^{*} 01 / 04$ & $37^{*} 01$ & CAVSEIGNTGKLIF & $2^{*} 01 / 2^{*} 02$ & $2^{*} 01$ & $2-3^{*} 01$ & CASSSRATDTQYF \\
\hline D8T106 & $8-4^{*} 01 / 04$ & $8^{*} 01$ & CVVSEGTGFQKLVF & $20-1^{*} 01 / 02 / 04 / 05 / 06$ & $1^{*} 01$ & $1-2^{*} 01$ & CSARVPQVYGYTF \\
\hline D8T107 & $25^{*} 01$ & $44^{*} 01$ & CAVYTGTASKLTF & $11-1^{*} 01$ & $2^{*} 01$ & $2-1^{*} 01$ & CASSFRPRGVNEQFF \\
\hline D8T108 & $8-6^{*} 02$ & $29 * 01$ & CAVRHNSGNTPLVF & $20-1^{*} / 04 / 05 / 06$ & $2^{*} 02$ & $2-2^{*} 01$ & CSARGSQGLAGTHTCELFF \\
\hline D8T114 & $\begin{array}{c}\text { AV14/DV4*02/03 } \\
12-3^{*} 01 / 02\end{array}$ & $\begin{array}{l}42^{*} 01 \\
52^{*} 01\end{array}$ & $\begin{array}{c}\text { CAGGGSQGNLIF } \\
\text { CAMSAGGTSYGKLTF }\end{array}$ & $6-5^{*} 01$ & $1^{*} 01$ & $1-3^{*} 01$ & CASSPSPPVPGSGNTIYF \\
\hline
\end{tabular}

${ }^{A}$ Described in a separate study (7). IMGT, International ImMunoGeneTics Information System.

In terms of binding to APCs, peptides comprising non-conforming $\mathrm{P} 1, \mathrm{P} 4, \mathrm{P} 6$, and $\mathrm{P} 9$ anchor residue substitutions (A25, A28, N28, R28, V28, R30, E33, and R33) demonstrated markedly reduced binding (specific binding <50\%) to DRA/DRB3*01:01-positive APCs (Figure 3). In contrast, peptides with substitutions expected to conform (I25, E28, T30, V30, I33, and V33) bound with about the same efficiency as the native L33 peptide.

Substitutions in "non-anchor" residues did not reduce binding to DRA/DRB3*01:01-positive APCs (STEINLIN); all demonstrated specific binding $>50 \%$. These residues, 26, 27, 29, 31, and 32, are all potentially in contact with TCR, as they are predicted to be solvent exposed.

In terms of $\mathrm{T}$ cell responsiveness (Table 4), all clones responded, as expected, to HPA-1a peptide (L33), although to various extents. In contrast, none reacted with LolP1, a peptide known to bind well to DRA/ DRB3*01:01 (9), and indeed, LolP1 bound well to DRB3*01:01-positive APCs (Figure 4). Furthermore, while most clones did not respond to autologous HPA-1b (P33) peptide, 3 clones responded weakly in some experiments but did not respond in others, and 1 clone, D8T106, responded consistently with moderate intensity in repeated experiments. Responses to peptides with substitutions in solvent-exposed residue were quite diverse, each peptide being efficient in stimulating some clones but not others. One exception was peptide L29; although binding well to APCs (Figure 4) this peptide did not activate any of the 14 clones (Table 4). One clone, D8T114, was sensitive to any change in the HPA-1a peptide, even to changes in residues 34 and 35, which are believed to extend beyond the peptide-binding groove of the DRA/DRB3*01:01 molecule. Together these data indicate that most HPA-1a-specific T cells recognize non-allogeneic integrin $\beta 3$ residues derived from HPA-1a-positive donors. Furthermore, these $\mathrm{T}$ cells are quite diverse with respect to the specific residues required for activation.

Autologous HPA-1b peptide at non-physiologically high concentrations can activate HPA-1a-specific T cells. Since HPA-1a-specific T cell clones recognize non-allogeneic residues, and, for most clones, not the Leu33 residue, the epitope recognized must also be present on the HPA-1b peptide. In support of this, 4 of the HPA-1a-specific T cell clones responded weakly to stimulation with HPA-1b peptide, possibly due to peptide densities exceeding physiological levels. To examine this closer, we tested whether we could increase the density of HPA-1b peptide on APCs by increasing peptide pulse concentrations and whether HPA-1a-specific T cells would respond to HPA-1b peptide in a dose-dependent manner.

Indeed, by increasing peptide pulse concentrations, levels of $\mathrm{P} 33$ peptide were increased on the APC surface in a dose-dependent manner (Figure 4). For comparison, similar experiments were performed with linker only, HPA-1a peptide (L33), and LolP1. Binding of L33 and LolP1 peptides also increased in a dose-dependent manner, and both of these peptides bound at higher densities to the APC surface com- 
Table 2. List of peptides used for stimulation of T cells.

\begin{tabular}{|c|c|c|c|}
\hline Antigen & Peptide & Amino acid sequence $^{A}$ & Peptide length \\
\hline HPA-1a & L33 (integrin $\beta 3$ 19-38) & CSPMCAWCSDEALPLGSPRC & 20-mer \\
\hline HPA-1b & P33 (integrin $\beta 3$ 19-38) & 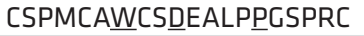 & 20-mer \\
\hline Control & LolP1 (191-210) & ESWGAVWRIDTPDKLTGPFT & 20-mer \\
\hline Control & LolP1 (196-208) & VWRIDTPDKLTG & 12-mer \\
\hline HPA-1a & L33 (integrin $\beta 3$ 24-35) & AWCSDEALPLGS & 12-mer \\
\hline \multirow[t]{29}{*}{ HPA-1b } & P33 (Integrin $\beta 3$ 24-35) & 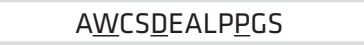 & 12-mer \\
\hline & $\mathrm{A} 25$ & 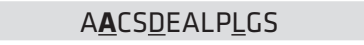 & 12-mer \\
\hline & 125 & AICSDEEALPLEGS & 12-mer \\
\hline & A26 & AWASDEEALPLGGS & 12-mer \\
\hline & $\mathrm{A} 27$ & AWCADEALPLGS & 12-mer \\
\hline & A28 & AWCS$\underline{\text { AEALPLGS }}$ & 12-mer \\
\hline & N28 & 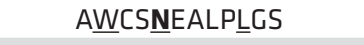 & 12-mer \\
\hline & E28 & AWㄷSEEEALPLLGS & 12-mer \\
\hline & $\mathrm{R} 28$ & AWCSREALPLGS & 12-mer \\
\hline & V28 & AWCSㅌEALPLGS & 12-mer \\
\hline & $\mathrm{D} 29$ & AWCSDDALPLGS & 12-mer \\
\hline & A29 & AWCSDAALPLGS & 12-mer \\
\hline & Q29 & AWCSDQQALPLGS & 12-mer \\
\hline & L29 & AWCSㅁALPLGS & 12-mer \\
\hline & T30 & AWCSDETLPLGS & 12-mer \\
\hline & R30 & AWCSDERLPLGS & 12-mer \\
\hline & V30 & AWCSDEVLPLGS & 12-mer \\
\hline & A31 & AWCSㅁAAPLEGS & 12-mer \\
\hline & V31 & AWCSDEEAVPLGS & 12-mer \\
\hline & A32 & AWCSDEEALALGS & 12-mer \\
\hline & V32 & AWCSDEALVLGS & 12-mer \\
\hline & 133 & AWCSDEALPIGS & 12-mer \\
\hline & V33 & AWCSDEALP哑S & 12-mer \\
\hline & E33 & AWCSDEALPEGGS & 12-mer \\
\hline & R33 & 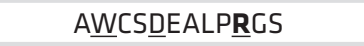 & 12-mer \\
\hline & A34 & AWCSDEALPLAS & 12-mer \\
\hline & W34 & AWCSDEALPLWWS & 12-mer \\
\hline & Y35 & AWCSDEALPLGY & 12-mer \\
\hline & A25-A28-A33 & A $\underline{A} C S \underline{A} E A L P \underline{A} C S$ & 12-mer \\
\hline
\end{tabular}

Identical peptide 12-mers with an N-terminal linker-biotin were used for peptide-binding assays. 20-mer peptides were used for stimulation of PBMC cultures from donors. All other experiments were performed with 12-mer peptides. AAnchor residues for DRA/DRB3*01:01 binding are underlined, and substituted amino acids are in bold.

pared with P33. When 4 different HPA-1a-specific T cell clones were assayed for activation with APCs pulsed with P33 concentrations above $5 \mu \mathrm{M}$, one of these, clone D48T12, was activated to secrete cytokines (Figure 5; only data for D48T12 are shown). This activation was antigen specific, since no activation was detected with even the highest peptide pulse concentration $(15 \mu \mathrm{M})$ of LolP1. In comparison, maximum activation was achieved with the lowest peptide-pulse concentration $(0.5 \mu \mathrm{M})$ of L33 peptide, and at $15 \mu \mathrm{M}$ or higher concentrations there were visible signs of $\mathrm{T}$ cell death by light microscopic examination (data not shown). Presumably, densities of L33 peptide on APCs with these conditions resulted in overstimulation and activation-induced cell death.

Thus, the epitope recognized by at least some HPA-1a-specific T cells must also be present on autologous HPA-1b peptide, since non-physiologically high concentrations could activate sensitive T cell clones.

\section{Discussion}

In the present study, the nature of alloantigen recognition by HPA-1a-specific T cells was examined. By careful analysis of 14 different clonal HPA-1a-specific $\mathrm{T}$ cell lines, we found predominantly that these do not directly recognize the allogeneic Leu33 residue. While this residue serves to stably bind HPA-1a peptide to 
Table 3. HLA class II defined B-LCLs as antigen-presenting cells.

\begin{tabular}{|c|c|c|c|c|c|c|}
\hline \multirow[b]{2}{*}{ Cell line } & \multicolumn{6}{|c|}{ HLA class II } \\
\hline & DRB3 & DRB1 & DQA1 & DQB1 & DPA1 & DPB1 \\
\hline STEINLIN (IHW\#9087) & *01:01 & ${ }^{*} 03: 01$ & ${ }^{*} 05: 01$ & ${ }^{*} 02: 01$ & ${ }^{*} 01$ & ${ }^{*} 03: 01,{ }^{*} 04: 01$ \\
\hline DUCAF (IHW\#9019)A & ${ }^{*} 02: 02$ & ${ }^{*} 03: 01$ & ${ }^{*} 05: 01$ & ${ }^{*} 02: 01$ & ${ }^{*} 01: 03$ & ${ }^{*} 02: 02$ \\
\hline EMJ (IHW\#9097) ${ }^{A}$ & *03:01 & *13:02 & *01:02 & *06:04 & ${ }^{*} 01$ & ${ }^{*} 03: 01,{ }^{*} 04: 01$ \\
\hline EK (IHW\#9054)A & ${ }^{*} 02: 02$ & *14:01 & ${ }^{*} 01: 04$ & ${ }^{*} 05: 03$ & ${ }^{*} 01: 03$ & ${ }^{*} 04: 02$ \\
\hline D4BL4 (in-house) & ${ }^{*} 01: 01,{ }^{*} 02: 02$ & ${ }^{*} 03: 01,{ }^{*} 14: 54$ & ${ }^{*} 05: 01, N D$ & ${ }^{*} 02: 01,{ }^{*} 05: 03$ & ND & ${ }^{*} 04: 01,{ }^{*} 32: 01$ \\
\hline
\end{tabular}

the DRA/DRB3*01:01 molecule $(9,10)$, our data show that this binding allows HPA-1a-specific T cells to recognize non-allogeneic residues in this peptide - residues that are in common with the HPA-1b peptide. The allogeneic Leu33 anchor residue itself seemingly does not form any structure detected by these $\mathrm{T}$ cells (with one possible exception). Without stable binding, which is the case with the HPA-1b peptide, HPA-1aspecific $\mathrm{T}$ cells are not activated, likely due to subthreshold densities of specific peptide-MHC complexes. In effect, HPA-1b peptide will be invisible to HPA-1a-specific T cells in HPA-1bb individuals, since it associates poorly with MHC. Furthermore, HPA-1a-specific T cell clones were heterologous with respect to their sensitivity to various point mutations in the peptide sequence. This suggests that several conformations formed by these non-allogeneic residues are recognized differently by different clones. This finding also demonstrates that the $\mathrm{T}$ cell response to these residues is diverse. Thus, the T cell response to HPA-1a represents a unique form of indirect allorecognition in which a non-allogeneic sequence becomes immunogenic by virtue of stable binding to MHC by an allogeneic anchor residue.

A noteworthy feature of the FNAIT-associated immune response is that anti-HPA-1a antibody production and severe FNAIT occurrence seem to be dependent on merely a single peptide-MHC complex: HPA-1a peptide bound to the DRA/DRB3*01:01 molecule. Although it cannot be ruled out that parallel $\mathrm{T}$ cell responses with other specificities (e.g., MHC class I-derived alloantigens) may be required for HPA-1a-specific antibody production, the strong association with the $D R B 3^{*} 01: 01$ allele (2-5), the stable binding of HPA-1a peptide to this MHC molecule $(9,10)$, and the isolation of HPA-1a-specific DRA/ $\mathrm{DRB}^{*}$ 01:01-restricted $\mathrm{CD} 4^{+} \mathrm{T}$ cells from HPA-1a-alloimmunized women $(7,8)$ lend support to the notion that other putative FNAIT-associated $\mathrm{T}$ cell responses likely play a minor role in immunization; alloimmune HPA-1a-specific antibody responses in DRB3*01:01-negative pregnant women are relatively rare $(2,3,6,12)$. Predictably, targeted manipulation of $\mathrm{T}$ cell recognition of the HPA-1a peptide:DRA/ DRB3*01:01 complex could be an effective mean to prevent or to reduce HPA-1a-specific antibody responses and thus prevent FNAIT occurrence. Toward this end, several studies have been aimed at the investigation of HPA-1a-specific T cell responses (13-15) and antigen processing and presentation (16). Several different $\mathrm{CD}^{+} \mathrm{T}$ cells specific for HPA-1a peptide were clonally isolated in 2 independent studies $(7,8)$. These respond by proliferation and cytokine secretion to stimulation with exogenous peptides containing the residue Leu33 but not Pro33, at relatively low and arguably physiologically relevant peptide concentrations. It was also shown that this recognition was restricted by the DRA/DRB3*01:01 molecule (7). Furthermore, HPA-1a-specific T cells were found to respond to autologous monocytes precultured with platelets from HPA-1a-positive but not HPA-1bb donors (7), demonstrating that physiologically relevant levels of processed antigen was readily recognized. Clonal HPA-1a-specific T cell lines were crucial for performing the current study and serve as an important tool for deciphering the immune response that leads to FNAIT and thus for identifying potential mechanisms that can be targeted to prevent FNAIT occurrence. In this study, peptide binding to APCs was measured instead of binding to isolated or synthetic MHC molecules. This was done to directly correlate peptide binding with $\mathrm{T}$ cell activation, as peptide binding efficiency to isolated or synthetic MHC molecules is not necessarily the same as binding to MHC molecules in the APC membrane. Integrin $\beta 3$ peptide binding to the DRA/DRB3*01:01 molecule has been characterized biochemically elsewhere $(9,10)$ and was not a focus of the current study.

Parallel assessment of $T$ cell activation and peptide-binding potential to DRB3*01:01-positive APCs in the present study demonstrated that peptide-binding efficiency was determining for $\mathrm{T}$ cell activation, 
A

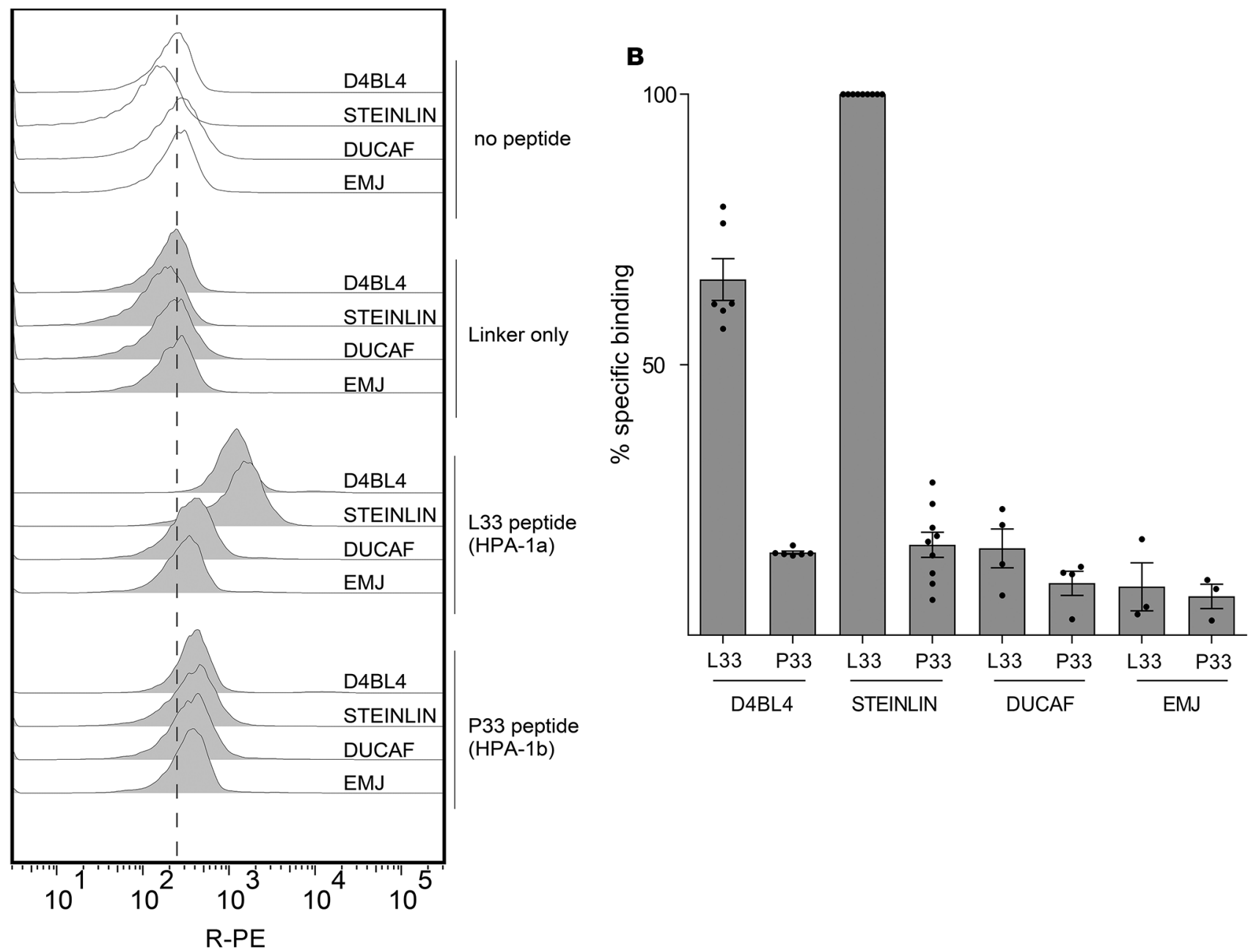

Figure 1. Peptide binding to cell lines. Binding of control 12-mer peptides ( 233 and P33) extended with a biotinylated linker peptide were incubated with DRB3*01:01-positive cell lines (STEINLIN and D4BL4) or control cell lines (DUCAF, EMJ) at $5 \mu \mathrm{M}$ peptide in the presence of $2.5 \mu \mathrm{M}$ AdEtOH. The efficiency of binding to APCs was assessed by flow cytometry with streptavidin-conjugated R-PE. (A) Representative raw data of one of 3 independent peptide binding assays (1 of 2 replicates shown). (B) Comparison of percent specific binding. Data points from independent experiments are presented as dots, with bars representing mean \pm SEM of at least 3 experiments. Raw data values were median R-PE fluorescence intensity on B-LCLs (gated by light scatter cytogram). Background intensity (cells only, no peptide) was subtracted before calculating the specific binding within each experiment (L33 peptide on STEINLIN defined as 100\%). AdEtOH, adamantane ethanol; APC, antigen presenting cell; R-PE, R-phycoerythrin; B-LCL, B-lymphoblastoid cell lines.

with HPA-1a versus HPA-1b peptides arguably representing the clearest example. In this respect, the small hydrophobic residues valine and isoleucine could substitute for Leu33, resulting in both efficient binding to MHC as well as T cell stimulation. Notably, a rare allelic integrin $\beta 3$ variant encoding Val33 instead of the common Leu33 and Pro33 variants has been identified (17). In the reported case, an HPA-1bb woman became alloimmunized in connection with pregnancy with an HPA-1a-negative but Val33-positive child, resulting in platelet-reactive antibodies and FNAIT. Arguably, the T cell stimulatory potential of this third HPA-1 variant, HPA-1c, was essential for antibody production and FNAIT onset in this pregnancy. In the current study, V33 peptide binds to APCs and stimulates HPA-1a-specific T cells as efficiently as L33 peptide. Thus, similar T cell specificities toward homologous residues would be expected in individuals immunized with HPA-1c. Although 13 of the $14 \mathrm{~T}$ cell clones examined in the current study responded equally to L33, V33, and I33 peptides, a recent study showed that "heteroclitic" peptides - with anchor residue modifications to improve binding - can discriminate TCR specificity for the original antigen due to structural alterations of the peptide-MHC complex $(18,19)$. This could be the case with the one exceptional clone examined in this study, D8T114, which responded to L33 but not to V33 and I33 peptides. We believe that the restricted mode of antigen recognition represented by 

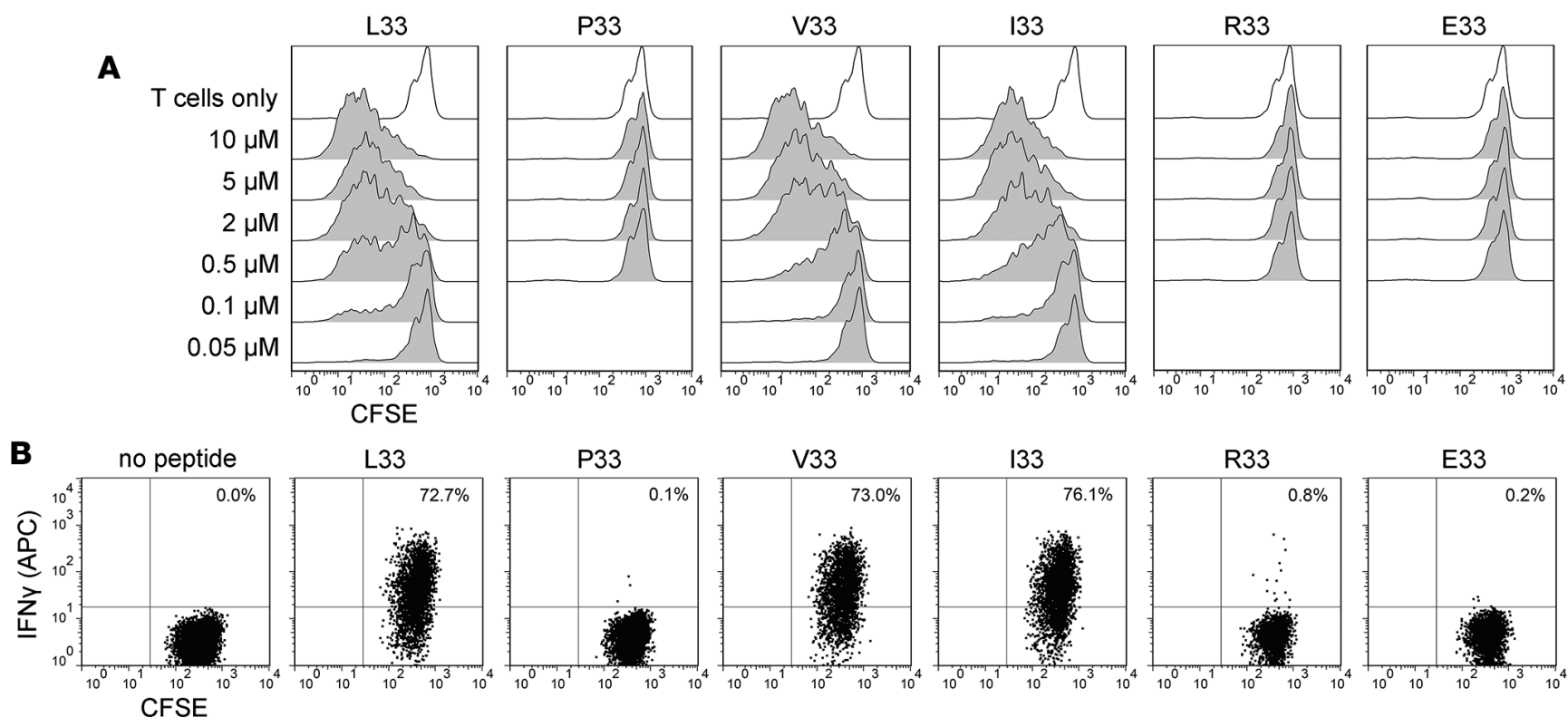

C

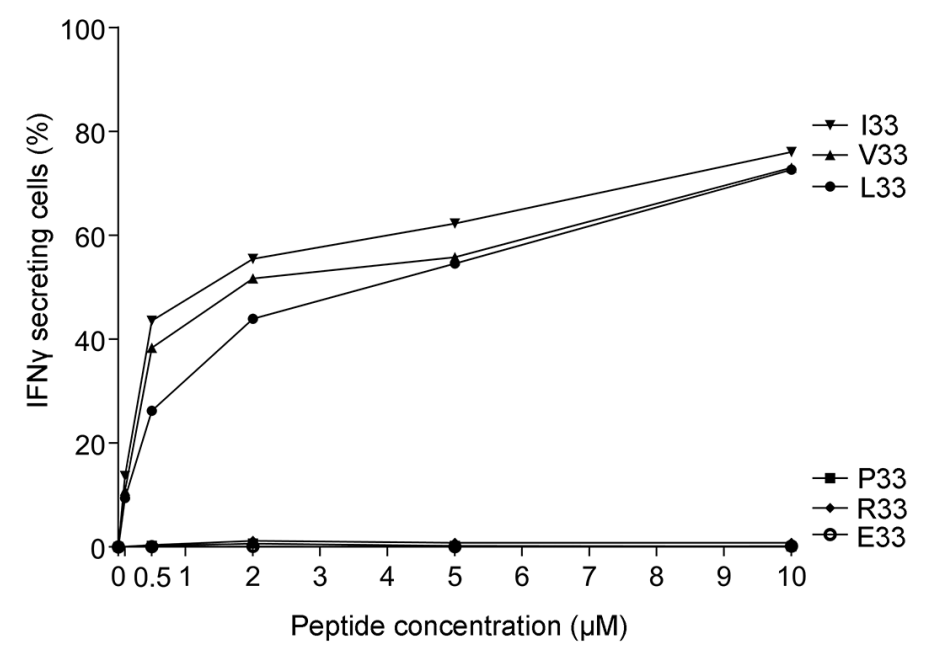

D

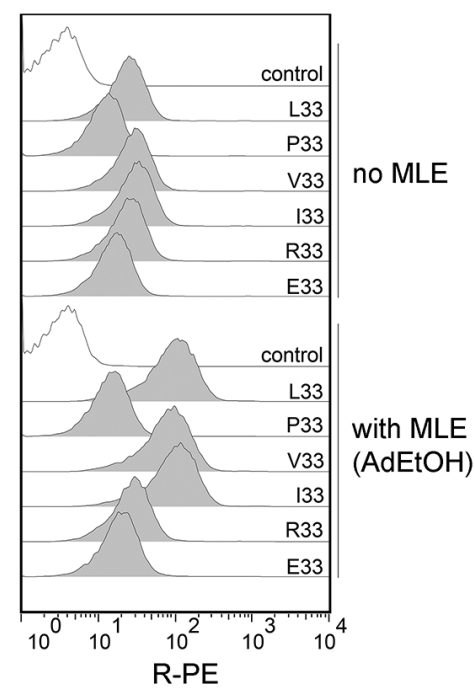

Figure 2. HPA-1a-specific T cells do not exclusively recognize the Leu33 residue in the HPA-1a peptide. (A) CFSE-labeled HPA-1a-specific T cell clones were stimulated with DRA/DRB3*01:01-positive APCs (D4BL4) pulsed with a panel of designed peptides in different concentrations, without MLE. T cell proliferation was determined after 7 days, illustrated by 1 of 4 experiments (D7T4). (B) T cell activation was also measured by intracellular cytokine staining (IFN- $\gamma$ ) by flow cytometry. T cells were gated by light scatter and CFSE staining. One representative of 3 experiments with $10 \mu \mathrm{M}$ peptide pulsing of D4BL4 cells, and (C) the effect of peptide pulsing concentrations on T cell activation. (D) Binding of peptides to D4BL4; same cell line used for T cell activation. Cells were pulsed with biotinylated peptides $(10 \mu \mathrm{M})$ with or without AdEtOH, stained with Streptavidin-R-PE, and analyzed in flow cytometry. The discrimination of peptide-binding efficiency is enhanced when using AdEtOH as an MLE. Substitutions of Leu33 to Arg/Clu reduced binding, while efficient binding was seen with substitutions with small, aliphatic residues Leu33 to Val/lle. Comparative binding experiments were conducted twice with 2 replicates; representative histograms are shown. Twelve-mer peptides were used in all experiments. HPA, human platelet antigen; MLE, MHC-loading enhancer; AdEtOH, adamantane ethanol.

this clone would not prevail in those individuals who become immunized with HPA-1c. Also, antigen recognition by this clone shows that the specificity of HPA-1a-specific T cells is not obvious; one could argue that since L33 serves as an anchor residue, T cell recognition would empirically be determined by other non-allogeneic residues.

In this study, we demonstrated that a relatively high concentration of HPA-1b peptide could activate HPA-1a-specific T cell clones. Similarly, Maslanka et al. (14) observed clonotypic T cell proliferation likely HPA-1a-specific T cells - in peripheral blood mononuclear cells (PBMCs) from HPA-1a-immunized women, cultured with equally high and even higher concentrations of a 13-mer integrin $\beta 3$ peptide (residues 26-38) that included Leu33 but lacked the Trp25 residue. Since Trp25 serves to stabilize binding 
Table 4. Activation of HPA-1a-specific clonal T cells.

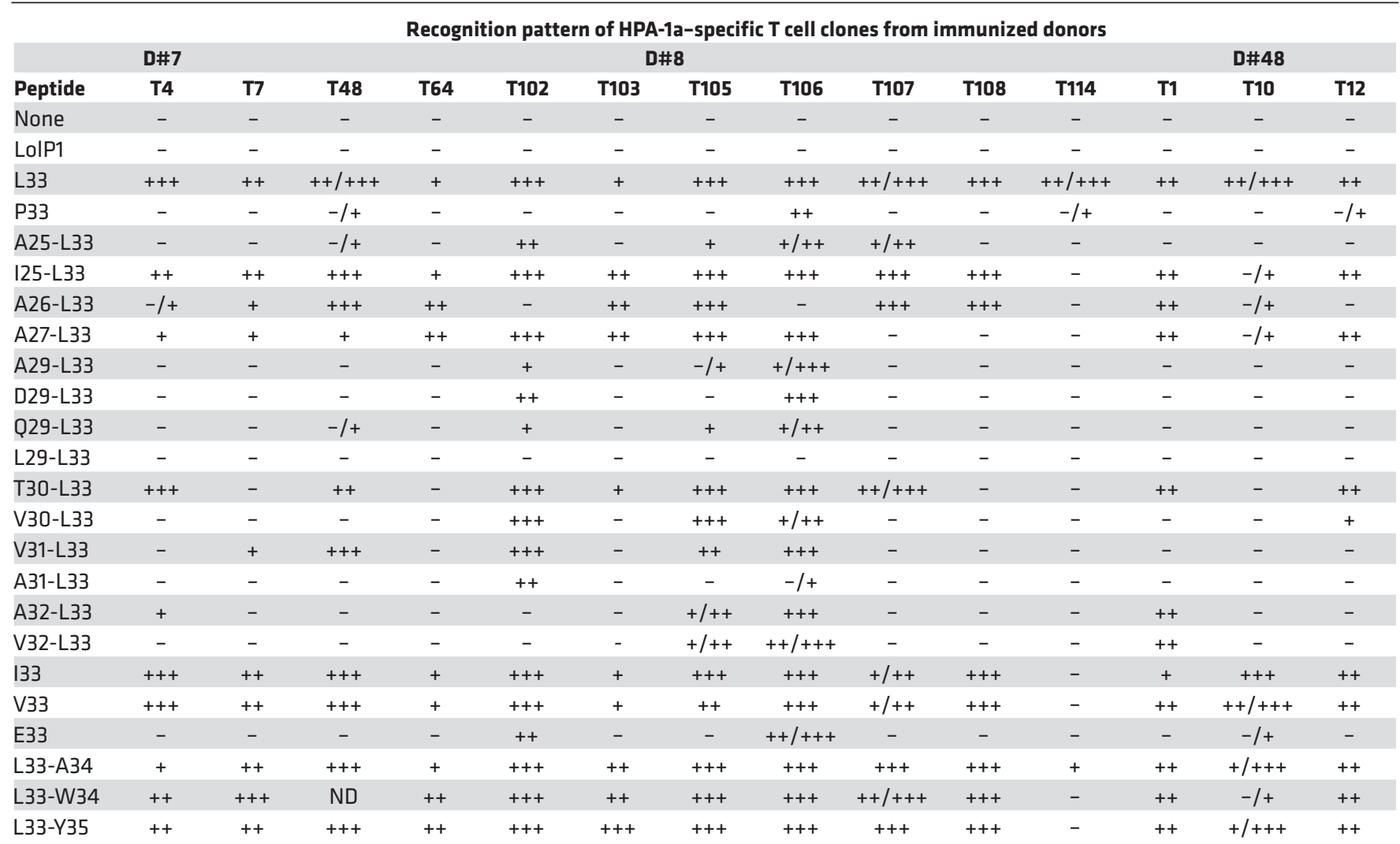

Screening of responsiveness of T cell clones when stimulated with DRA/DRB3*01:01-positive APCs (D4BL4) pulsed with modified peptides (12-mers).

T cells were gated by light scatter and CFSE staining. Responsiveness was measured by TNF- $\alpha$ secretion. T cell responses designations:,$- \leq 5 \%$ (no response); $+, 5 \% \leq 40 \%,++, 40 \% \leq 80 \%$; and +++, >80\%. (+) designates weak response in some experiments. ND, no data available. In the screening experiment, all reactions were run once, and repeated experiments were performed with most reactions to verify the pattern. T cell responsiveness to different peptides was consistent in repeated experiment, with only small variations with some clones; this is indicated by 2 different scores separated by a slash.

of HPA-1a peptide to DRA/DRB3*01:01, one can predict that this peptide would not bind well to MHC. For perspective, a similar high concentration of HPA-1a peptide was lethal to the same T cells (data not shown), likely due to activation-induced cell death by high densities of peptide-MHC. It is possible that the absence of the Trp25 anchor was compensated by increased peptide concentrations in the noted study. It should be mentioned that the same research group later identified the peptide-binding motif of the DRA/ DRB3*01:01 molecule and the 3 anchor residues determining for stable binding of HPA-1a peptide to this MHC molecule (9). In view of this, similar clonotypic proliferative responses would likely have been achieved at considerably lower peptide concentrations had the stimulating peptide been extended by a single amino acid in the $\mathrm{N}$-terminus to include the third anchor residue.

A noteworthy observation in our study is that several clones displayed slight reactivity toward autologous HPA-1b peptide. While the donors had no signs of autoimmunity, it is possible that autoreactivity toward HPA-1b peptide set the stage for responses to the shared epitope on HPA-1a peptide. One could speculate that HPA-1b peptide has a role in thymic selection, selecting $\mathrm{T}$ cells that are tolerant to peripheral HPA- $1 \mathrm{~b}$ and that respond to stably bound HPA-1a peptide in connection with HPA-1-incompatible pregnancy. On the other hand, the autoreactivity could also be due to experimental peptide densities close to the threshold for self-tolerance.

As noted above, our findings demonstrate that the integrin $\beta 3$-derived epitope common to both HPA- 1 variants is recognized by HPA-1a-specific T cells. Arguably, this may allow for a more diverse $\mathrm{T}$ cell response than if the immunogenic epitope was limited to the allogeneic Leu33 residue alone. Indeed, this can also explain why no obvious conserved TCR $\alpha / \beta$ CDR3 motifs are seen in TCR s of the T cell clones. 


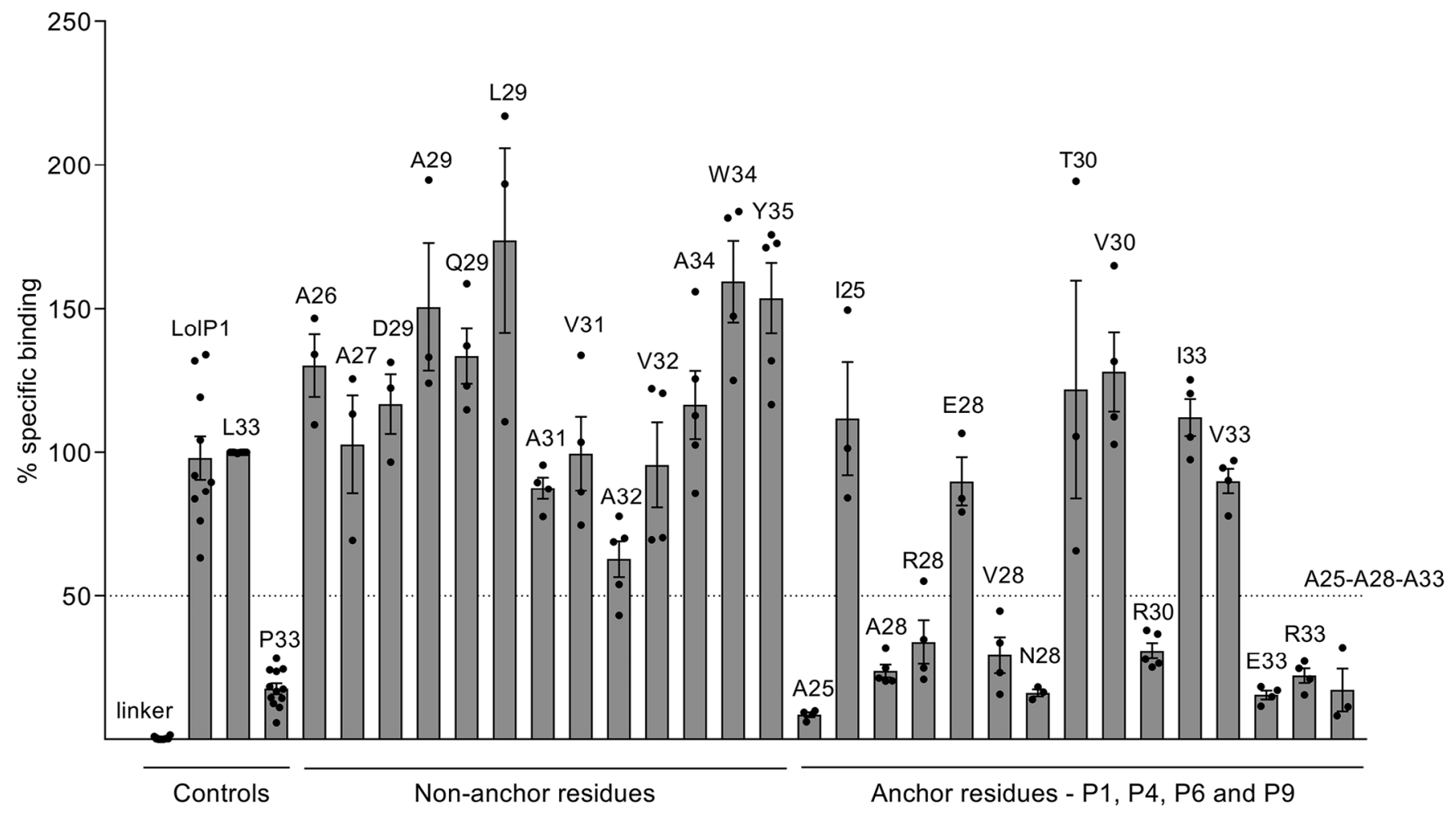

Figure 3. Peptide binding to the DRA/DRB3ai*01:01-positive cell line STEINLIN. Peptides (12-mers) with single or multiple amino acid substitutions compared with HPA-1a peptide, and extended with a biotinylated linker peptide, were incubated with STEINLIN cells at $5 \mu \mathrm{M}$ and $10 \mu \mathrm{M}$ peptide in the presence of $2.5 \mu \mathrm{M}$ AdEtOH. The efficiency of binding to APCs was assessed by flow cytometry with streptavidin-conjugated R-PE. Binding was measured as percent specific binding (L33 peptide defined as 100\%). Each peptide that bound efficiently to STEINLIN was also examined for binding to MHC-matched cell lines DUCAF, EMJ, and EK such that binding to any other MHC class II molecule on STEINLIN could be ruled out. Data points from independent experiments are presented as dots, with bars representing mean \pm SEM of at least 3 experiments. Raw data values were median R-PE fluorescence intensity on B-LCLs (gated by light scatter cytogram). Background intensity (cells only, no peptide) was subtracted before calculating the specific binding within each experiment. AdEtOH, adamantane ethanol; APC, antigen-presenting cell; R-PE, R-phycoerythrin; B-LCL, B-lymphoblastoid cell lines

An interesting question is how the quality of the $\mathrm{T}$ cell response will shape the quality of corresponding anti-HPA-1a antibody responses in relation to strategies to modulate HPA-1a-specific T cell responses. The data presented herein will potentially be useful in this respect. One strategy will be to induce immune tolerance to HPA-1a by oral administration of peptides in a tolerogenic formulation. Several of the peptides examined in this study will be tested for this. Important qualities will be efficient binding to the HLA-DRA/DRB3*01:01 molecule and recognition by most HPA-1a-specific T cells. In addition to the L33 peptide, both the V33 and I33 peptides have these characteristics. Tolerance induction may depend on induction of tolerogenic regulatory $\mathrm{T}$ cells, although it could also depend on clonal deletion or induction of clonal anergy (20). Therefore, tolerance induction with such native or engineered peptides could be sufficient to prevent formation of anti-HPA1a antibodies. The $\mathrm{T}$ cell clones employed in this study were isolated from 3 women. While the time from delivery to blood collection differed considerably, the specificities of the $\mathrm{T}$ cells have likely been preserved.

In conclusion, we have demonstrated that $\mathrm{T}$ cell responses to HPA-1a are diverse and involve a unique form of indirect allorecognition in which non-allogeneic residues - common to both fetus and mother - are recognized, while the allogeneic Leu33 residue, which serves to bind the peptide stably to MHC, is not directly recognized. These findings may have implications for development of antigen-specific prophylaxis to prevent $\mathrm{T}$ cell responses that underlie FNAIT occurrence. In addition, it is possible that this form of allorecognition could be a recognition mechanism involved in other systems such as tissue rejection responses following organ transplantation and those resulting in transfusion refractoriness.

\section{Methods}

Human donors. Blood from 3 HPA-1bb, HLA-DRB3*01:01-positive women with anti-HPA-1a alloantibodies was used. The clone isolated from donor 7 was described in a previous study (7). Donor 7 was a 32-year-old 


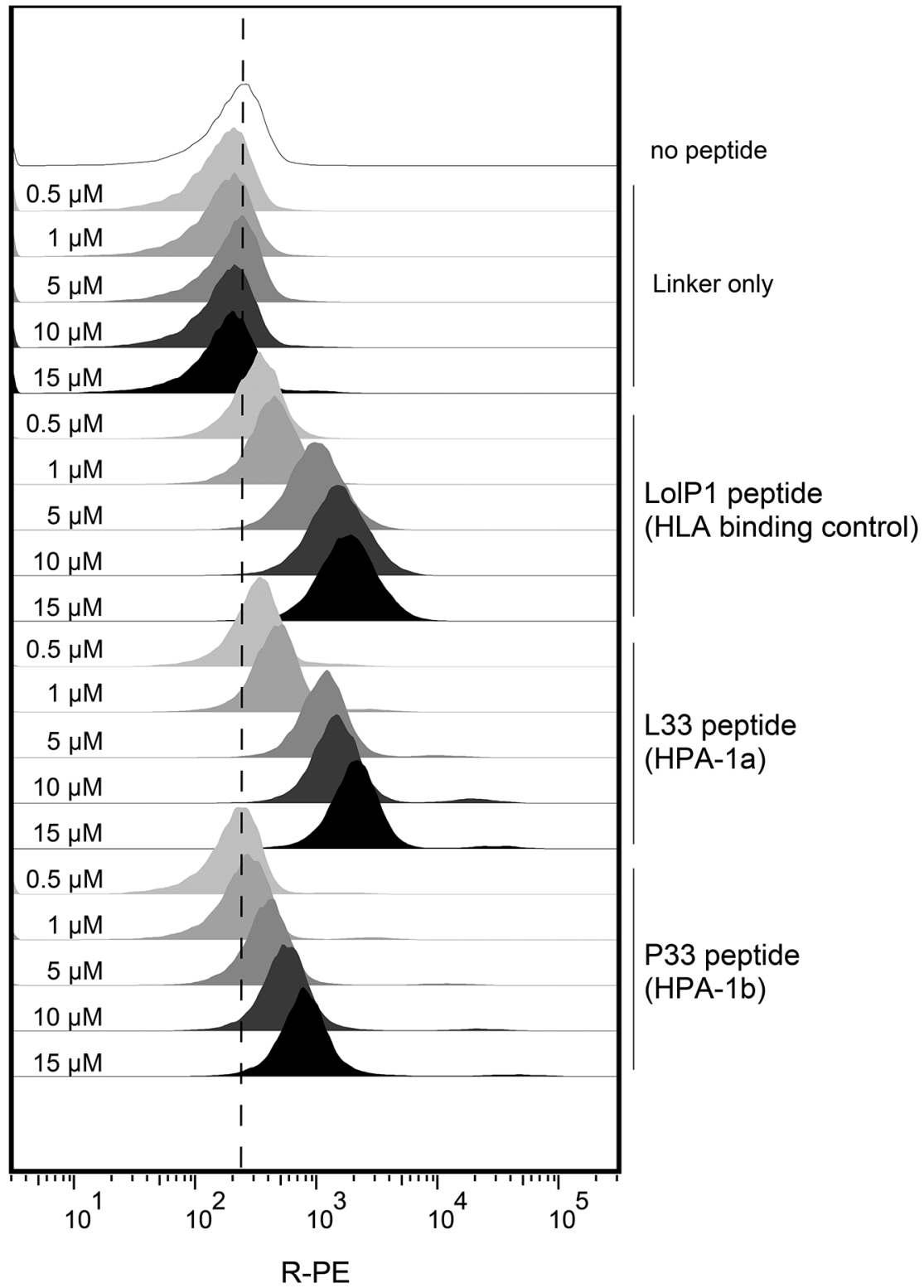

Figure 4. Peptide binding is dose dependent. Binding of control 12-mer peptides (linker only, L33, and P33) to HLA-DRB3*01:01-positive APCs (D4BL4), measured by flow cytometry after peptide pulsing with AdEtOH as MLE. No increase was seen with linker only, while an increase can be seen with L33, LolP1, and P33. Experiments were conducted twice with two replicates; representative histograms are shown. AdEtOH, adamantane ethanol; APC, antigen-presenting cell; MLE, MHC-loading enhancer.

who gave birth to a severely thrombocytopenic, HPA-1ab child (platelet count $9 \times 10^{9} / 1$ ) in 2007 . The child presented with petechiae and intracerebral bleedings. Maternal anti-HPA-1a antibodies were barely detectable at delivery. Seven weeks postpartum (when the blood sample was collected), low levels of maternal anti-HPA-1a antibody were detected (1.25 IU/ml). Donor 8 was a 54-year-old (at blood collection in 2010) who had given birth to a severely thrombocytopenic child (platelet count $<10 \times 10^{9} / 1$, HPA-1ab) in 1980 . The anti-HPA-1a in maternal plasma was $\sim 30 \mathrm{IU} / \mathrm{ml}$ in 2008 and 2010. Donor 48 was a 41-year-old who had given birth to 2 severely thrombocytopenic, HPA-1ab children; one in 1999 (platelet count <10 × 109/1; maternal anti-HPA-1a was $37 \mathrm{IU} / \mathrm{ml}$ at delivery) and another in 2003 (platelet count $87 \times 10^{9} / 1$; maternal anti-HPA-1a was $17 \mathrm{IU} / \mathrm{ml}$ at delivery). The level was $108 \mathrm{IU} / \mathrm{ml}$ in the buffy coat donated in 2008, which was used for isolation of cells in this study. The anti-HPA-1a antibody levels were determined during $2007-$ 2010 by a quantitative monoclonal antibody immobilization of platelet antigen (MAIPA) assay (21) by the 
A

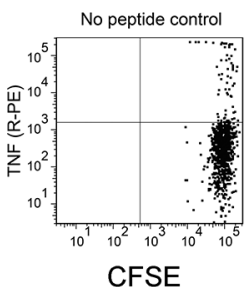

L33

P33
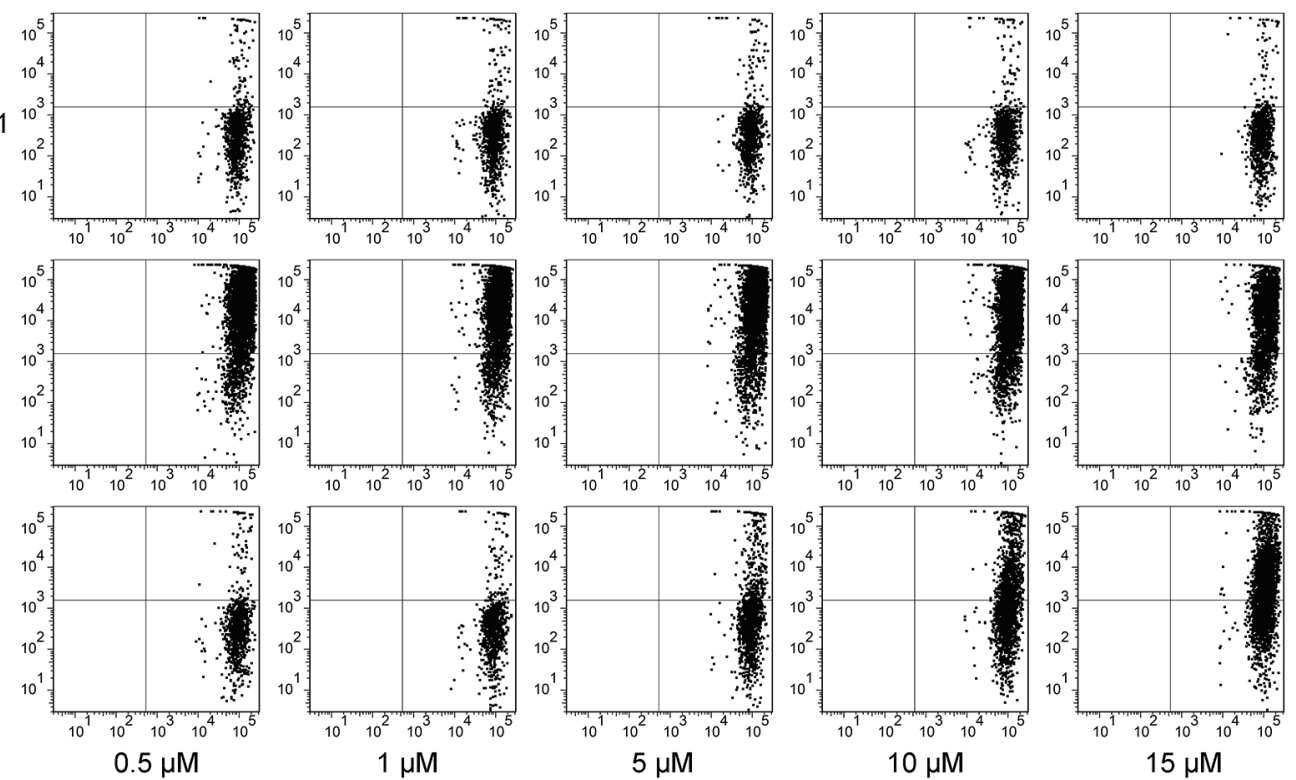

$\mathbf{C}$
B

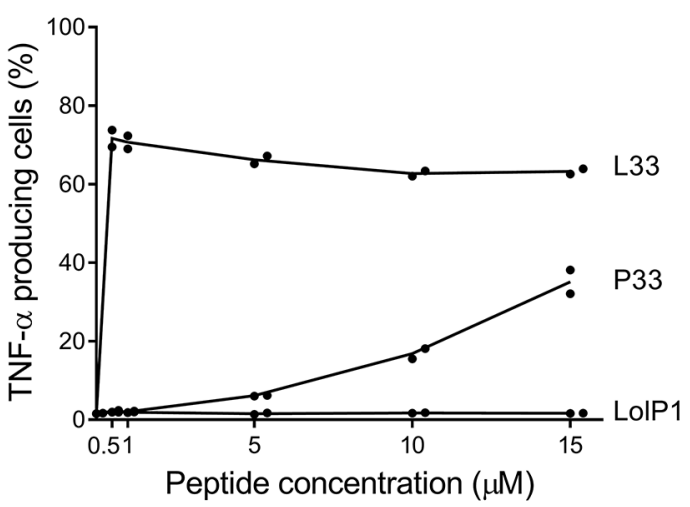

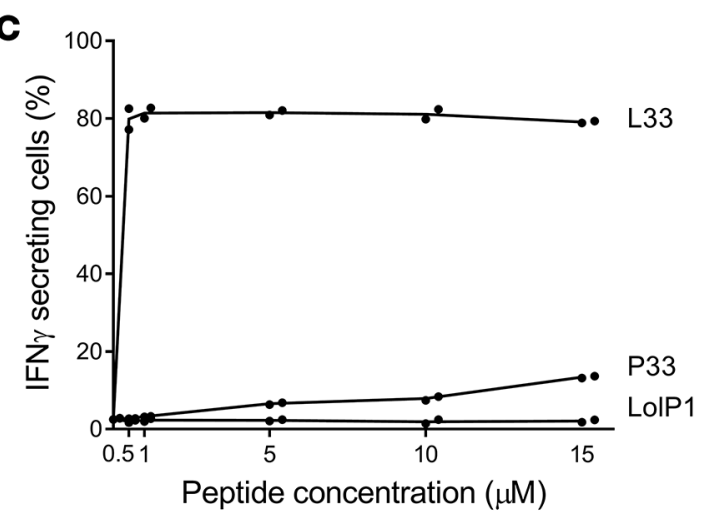

Figure 5. High concentrations of P33 can also activate T cell clones. T cell activation measured by (A and B) TNF- $\alpha$ secretion and (C) IFN- $\gamma$ secretion with clone D48T12 upon stimulation with APCs pulsed with increasing 12-mer peptide concentrations. T cells were gated by light scatter and CFSE staining. Experiments were conducted twice with 2 replicates; representative results are shown. APC, antigen-presenting cell

Norwegian National Unit for Platelet Immunology (University Hospital of North Norway). The MAIPA is routinely calibrated against a polyclonal anti-HPA-1a standard from the UK National Institute of Biological Standards and Control (22).

Handling of blood samples. PBMCs from $50 \mathrm{ml}$ buffy coat (donor 8) or $30 \mathrm{ml}$ peripheral blood (donor 48) were isolated by density gradient separation within 30 hours (7). Cells were cryopreserved $\left(10^{7}\right.$ cells/vial) in $10 \%$ DMSO, 90\% heat-inactivated FBS (Lonza), and stored in liquid nitrogen ( $<2$ years). Frozen cells were rapidly thawed at $50^{\circ} \mathrm{C}$ and washed immediately in cold complete media (CM) consisting of Iscove's modified Dulbecco's medium (IMDM, Lonza), 10\% FBS, 4\% HPA-1bb human serum, and penicillin/streptomycin (Lonza). Thawed cells were rested overnight, and viable cells were collected by density gradient. In one experiment, fresh donor 8 PBMCs were used. All cell cultures were kept at $37^{\circ} \mathrm{C}$ in a $7.5 \% \mathrm{CO}_{2}$ humidified atmosphere.

Synthetic peptides. 12-mer peptides, 12-mer peptides with an N-terminal biotinylated linker (biotin-KSGGGSGGGSGGGSGG-peptide; Biotechnology Centre of Oslo, University of Oslo, Norway), and 20-mer peptides (Eurogentec) were dissolved in $20 \mu \mathrm{DMSO}$, diluted to $1 \mathrm{ml}$ in sterile water, further diluted to 88 $\mu \mathrm{M}$ stock solutions in $0.2 \% \mathrm{BSA}$ in PBS $(0.2 \% \mathrm{PBSA})$, and stored at $-20^{\circ} \mathrm{C}$.

Isolation of HPA-1a-specific $\mathrm{T}$ cell clones. Clonal HPA-1a-specific $\mathrm{T}$ cells were isolated as previously described (7) with some modifications. Briefly, synthetic HPA-1a antigen (20-mer peptides; Table 2) or native antigen (HPA-1a-positive platelets) with corresponding controls were used to stimulate donor PBMCs. Sin- 
gle cell sorting of activated cells (proliferating or TNF- $\alpha$-producing cells) was performed from antigen-specific stimulation cultures on a FACSAria (BD Biosciences). Sorted cells were expanded and specificity-tested by ELISPOT assays and maintained in culture as previously described (7). Thirteen unique HPA-1a-specific clonal T cell cultures were established, in addition to D7T4. Six were established from single cell sorting of $\mathrm{CD}^{+}$proliferating cells in CFSE proliferation assays with peptide antigen (20-mer L33, $\left.2.5 \mu \mathrm{M}\right)$ from PBMCs after 8 days; from donor 8: D8T7, D8T64, D8T105 and donor 48: D48T1, D48T10, D48T12. Six were established from single cell sorting of $\mathrm{CD} 4^{+} \mathrm{TNF}-\alpha$-secreting cells following a 4.5-hour peptide stimulation (20-mer L33, $2 \mu \mathrm{M})$ at $37^{\circ} \mathrm{C}$ of donor 8 PBMCs in the presence of anti-human TNF- $\alpha(1: 100$, R-phycoerythrin [R-PE], clone MAb11) and $10 \mu \mathrm{M}$ TAPI-0 (Merck): D8T102, D8T103, D8T105, D8T106, D8T107, D8T108. Finally, 2 were identified by the platelet-stimulated donor 8 cultures: D8T48, D8T114.

$T$ cell receptor gene analyses. Total RNA was isolated from $10^{6}$ clonal T cells using an RNeasy Mini Kit (QIAGEN). cDNA was synthesized from $500 \mathrm{ng}$ total RNA by Superscript Reverse Transcriptase III (Invitrogen) and random primers (Promega). TRAV/TRBV was determined by the same strategy and cycling programs as previously described $(7,23)$ using QuantiTect SYBR Green PCR kit (QIAGEN), on an ABI PRISM 7900HT (Applied Biosystems) with $T R B V$-specific $(23,24)$ and $T R A V$-specific (25) primer sets. Results were analyzed using Sequence Detection Systems 2.2.2 software (Applied Biosystems). Sequencing was performed after determination of the appropriate $T R A V$ and $T R B V$ primers for a given clone (7).

Flow cytometry. Anti-human CD4 (R-PE-Alexa Fluor 610, clone S3.5) and anti-human IFN- $\gamma$ (APC, clone B27) antibodies were from Molecular Probes. Anti-human TNF- $\alpha$ antibody (R-PE, clone MAb11) was from BD Biosciences. All samples were washed in $0.2 \%$ PBSA prior to flow cytometry, performed on a FACS Canto II with FACSDiva version 6 software (BD Biosciences). Photomultiplier tube (PMT) voltages were set using BD CS\&T beads. Compensation was calculated using single stained samples. Data were analyzed using FlowJo7.5 software (TreeStar).

APCs. HLA class II-defined EBV-transformed B-LCLs STEINLIN, DUCAF, EMJ, and EK were obtained from the International Histocompatibility Working Group (IHWG) through the Fred Hutchinson Cancer Research Center (Seattle, Washington, USA). In addition, an in-house EBV-transformed B-LCL, D4BL4, from an HPA-1bb donor was used. HLA class II typing for this cell line was performed by the Norwegian Bone Marrow Registry, Oslo, Norway. All B-LCLs used in this study (Table 3) were cultured in IMDM, 10\% FBS, and penicillin-streptomycin and regularly tested for mycoplasma contamination.

Peptide pulsing of B-LCLs. B-LCLs were pulsed with $0.05-15 \mu \mathrm{M}$ peptide in CM for 3.5 hours at $37^{\circ} \mathrm{C}$ as a standard protocol. In experiments to determine peptide binding and the corresponding reactivity pattern of the different $\mathrm{T}$ cell clones, $2.5 \mu \mathrm{M}$ AdEtOH (Sigma-Aldrich) was used as an MLE. After pulsing, cells were washed twice in $0.2 \%$ PBSA and once in CM to ensure minimal rest of unbound peptide. For peptide-binding assays, APCs were pulsed with biotinylated peptides, stained with Streptavidin-R-PE (Molecular Probes), and washed in $0.2 \% \mathrm{PBSA}_{0} 0.02 \% \mathrm{NaN}_{3}$ prior to flow cytometry.

$T$ cell activation assays. Clonal $\mathrm{T}$ cells in long-term cultures were CFSE-stained and rested overnight in CM without IL-2 prior to experiments to ensure high viability (>85\%) and a resting status. Clonal $\mathrm{T}$ cells and peptide-pulsed B-LCLs were cocultured (5:3 ratio) in 200- $\mu$ l reactions.

Production of IFN- $\gamma$ was measured by intracellular cytokine staining after coculture of T cells and peptide-pulsed B-LCLs for 12 hours in CM. Brefeldin A (5 $\mu \mathrm{g} / \mathrm{ml}$; Sigma-Aldrich) was added after 90 minutes' incubation. Cells were washed and fixed in PBS 4\% paraformaldehyde for 15 minutes and permeabilized in HEPES/HBSS $0.1 \%$ saponin for 10 minutes. Cells were stained for 15 minutes at room temperature by adding anti-human IFN- $\gamma(1: 100 ;$ APC). Production of TNF- $\alpha$ was measured by a surface-staining protocol (26). Briefly, CFSE-stained T cells and peptide-pulsed B-LCLs were cocultured in CM for 4.5 hours with anti-human TNF- $\alpha$ (1: 200; R-PE) and $10 \mu \mathrm{M}$ TAPI-0 (Merck), washed, and analyzed immediately by flow cytometry. Proliferation was assayed by measuring CFSE intensity after 7-day coculture of $\mathrm{T}$ cells with peptide-pulsed B-LCLs (irradiated 12,000 rad), supplemented with IL-2 (30 U/ml) and IL-15 (5 ng/ $\mathrm{ml}$ ) on day 2. Cells were stained for 15 minutes at room temperature with anti-human CD4 (1:200; R-PEAlexa Fluor 610), washed, and analyzed by flow cytometry.

Statistics. Statistical analysis and graphs were made using Prism 5 (GraphPad Software Inc.). For all plots, error bars show mean \pm SEM.

Study approval. The study was approved by the Regional Committee for Medical Research Ethics (REC North), University of Tromsø - The Arctic University of Norway. Blood samples were drawn from donors after written informed consent was obtained in accordance with the Declaration of Helsinki. 


\section{Author contributions}

MTA and TBS designed the study, analyzed the data, and wrote the manuscript. MTA and ILK performed the experiments. AH and BS contributed with conceptual input and revised drafts of the manuscript. All authors took part in constructive discussions throughout the study and critically reviewed the final version of the manuscript.

\section{Acknowledgments}

This work was supported by grants from the Northern Norway Regional Health Authority (MTA) and by the Research Council of Norway (191437). We thank Andreas Tendeland Uhre for technical assistance with peptide-binding experiments and the Blood Bank and the Norwegian National Unit for Platelet Immunology at the University Hospital of North Norway for providing blood products and for quantitation of anti-HPA-1a antibody in plasma samples. We also thank the Norwegian Bone Marrow Registry for HLA typing and the Bioimaging Core Facility, University of Tromsø - The Arctic University of Norway, for use of the FACS Aria cell sorter.

Address correspondence to: Tor Brynjar Stuge, Immunology Research Group, Department of Medical Biology, University of Tromsø - The Arctic University of Norway, MH-building, N-9037 Tromsø, Norway. Phone: 47.77626312; E-mail: tor.brynjar.stuge@uit.no.

1. Newman PJ, Derbes RS, Aster RH. The human platelet alloantigens, P1A1 and P1A2, are associated with a leucine33/ proline33 amino acid polymorphism in membrane glycoprotein IIIa, and are distinguishable by DNA typing. JClin Invest. 1989;83(5):1778-1781

2. Kjeldsen-Kragh J, et al. A screening and intervention program aimed to reduce mortality and serious morbidity associated with severe neonatal alloimmune thrombocytopenia. Blood. 2007;110(3):833-839.

3. Williamson LM, et al. The natural history of fetomaternal alloimmunization to the platelet-specific antigen HPA-1a (P1A1, Zwa) as determined by antenatal screening. Blood. 1998;92(7):2280-2287.

4. Valentin N, et al. HLA-DRw52a is involved in alloimmunization against PL-A1 antigen. Hum Immunol. 1990;27(2):73-79.

5. L'Abbé D, et al. Alloimmunization to platelet antigen HPA-1a (PIA1) is strongly associated with both HLA-DRB3*0101 and HLA-DQB1*0201. Hum Immunol. 1992;34(2):107-114.

6. Maslanka K, Guz K, Zupanska B. Antenatal screening of unselected pregnant women for HPA-1a antigen, antibody and alloimmune thrombocytopenia. Vox Sang. 2003;85(4):326-327.

7. Ahlen MT, Husebekk A, Killie MK, Skogen B, Stuge TB. T-cell responses associated with neonatal alloimmune thrombocytopenia: isolation of HPA-1a-specific, HLA-DRB3*0101-restricted CD4+ T cells. Blood. 2009;113(16):3838-3844.

8. Rayment R, et al. Evidence for the specificity for platelet HPA-1a alloepitope and the presenting HLA-DR52a of diverse antigen-specific helper T cell clones from alloimmunized mothers. J Immunol. 2009;183(1):677-686.

9. Wu S, Maslanka K, Gorski J. An integrin polymorphism that defines reactivity with alloantibodies generates an anchor for MHC class II peptide binding: a model for unidirectional alloimmune responses. J Immunol. 1997;158(7):3221-3226.

10. Parry CS, Gorski J, Stern LJ. Crystallographic structure of the human leukocyte antigen DRA, DRB3*0101: models of a directional alloimmune response and autoimmunity. J Mol Biol. 2007;371(2):435-446.

11. Dickhaut K, et al. Enhancement of tumour-specific immune responses in vivo by 'MHC loading-enhancer'(MLE). PLoS One. 2009;4(9):e6811.

12. Turner ML, et al. Prospective epidemiologic study of the outcome and cost-effectiveness of antenatal screening to detect neonatal alloimmune thrombocytopenia due to anti-HPA-1a. Transfusion. 2005;45(12):1945-1956.

13. Jackson DJ, Murphy MF, Soothill PW, Lucas GF, Elson CJ, Kumpel BM. Reactivity of T cells from women with antibodies to the human platelet antigen (HPA)-1a to peptides encompassing the HPA-1 polymorphism. Clin Exp Immunol. 2005;142(1):92102.

14. Maslanka K, Yassai M, Gorski J. Molecular identification of T cells that respond in a primary bulk culture to a peptide derived from a platelet glycoprotein implicated in neonatal alloimmune thrombocytopenia. J Clin Invest. 1996;98(8):1802-1808.

15. Sukati H, Bessos H, Barker RN, Urbaniak SJ. Characterization of the alloreactive helper T-cell response to the platelet membrane glycoprotein IIIa (integrin-beta3) in human platelet antigen-1a alloimmunized human platelet antigen-1b1b women. Transfusion. 2005;45(7):1165-1177.

16. Anani Sarab G, Moss M, Barker RN, Urbaniak SJ. Naturally processed peptides spanning the HPA-1a polymorphism are efficiently generated and displayed from platelet glycoprotein by HLA-DRB3*0101-positive antigen-presenting cells. Blood. 2009;114(9):1954-1957.

17. Santoso S, et al. A naturally occurring LeuVal mutation in beta3-integrin impairs the HPA-1a epitope: the third allele of HPA-1. Transfusion. 2006;46(5):790-799.

18. Madura F, et al. Structural basis for ineffective T-cell responses to MHC anchor residue-improved "heteroclitic" peptides. Eur $J$ Immunol. 2015;45(2):584-591.

19. Cole DK, et al. Modification of MHC anchor residues generates heteroclitic peptides that alter TCR binding and T cell recognition. J Immunol. 2010;185(4):2600-2610.

20. Weiner HL, da Cunha AP, Quintana F, Wu H. Oral tolerance. Immunol Rev. 2011;241(1):241-259. 
21. Bertrand G, et al. Quantification of human platelet antigen-1a antibodies with the monoclonal antibody immobilization of platelet antigens procedure. Transfusion. 2005;45(8):1319-1323.

22. Allen D, et al. Collaborative study to establish the first international standard for quantitation of anti-HPA-1a. Vox Sang. 2005;89(2):100-104.

23. Lee KH, et al. Functional dissociation between local and systemic immune response during anti-melanoma peptide vaccination. J Immunol. 1998;161(8):4183-4194.

24. Akatsuka Y, Martin EG, Madonik A, Barsoukov AA, Hansen JA. Rapid screening of T-cell receptor (TCR) variable gene usage by multiplex PCR: application for assessment of clonal composition. Tissue Antigens. 1999;53(2):122-134.

25. Yao XS, Diao Y, Sun WB, Luo JM, Qin M, Tang XY. Analysis of the CDR3 length repertoire and the diversity of TCR alpha chain in human peripheral blood T lymphocytes. Cell Mol Immunol. 2007;4(3):215-220.

26. Haney D, et al. Isolation of viable antigen-specific CD8+ T cells based on membrane-bound tumor necrosis factor (TNF)- $\alpha$ expression. J Immunol Methods. 2011;369(1-2):33-41. 